\title{
THE BASE COMPONENTS OF THE DUALIZING SHEAF OF A CURVE ON A SURFACE
}

\author{
KAZUHIRO KONNO AND MARGARIDA MENDES LOPES
}

\begin{abstract}
This note studies the structure of the divisorial fixed part of $\left|\omega_{D}\right|$ for a 1-connected curve $D$ on a smooth surface $S$. It is shown that if the divisorial fixed part $F$ of $\left|\omega_{D}\right|$ is non empty then it has arithmetic genus $\leq 0$ and each component of $F$ is a smooth rational curve. The stucture of curves $D$, with non empty divisorial fixed part $F$ for $\left|\omega_{D}\right|$, is also described.
\end{abstract}

\section{INTRODUCTION}

In this note we study the structure of the fixed part of $\left|\omega_{D}\right|$ for a 1-connected curve $D$ on a smooth surface $S$. It is well known that if $\left|\omega_{D}\right|$ has base points then $D$ is not 2-connected (cf. [CFM]), and in fact there has been work of several authors concerning the structure of $\omega_{D}$ (see, e.g., [CCFR, [CFM], $[\mathrm{K}], \mathrm{M}]$ ) but as far as we know the present result is new.

We prove the following theorem:

Theorem 0.1. Let $D$ be a 1-connected curve on a smooth algebraic projective surface $S$ over $\mathbb{C}$. If $0 \prec Z \prec D$ is a curve contained in the fixed part of $\left|\omega_{D}\right|$ then:

i) every irreducible component of $Z$ is a smooth rational curve;

ii) for any $0 \prec Z^{\prime} \preceq Z, p_{a}\left(Z^{\prime}\right) \leq 0$ and $h^{1}\left(Z^{\prime}, \mathcal{O}_{Z^{\prime}}\right)=0$;

iii) $p_{a}(Z)=0$ if and only if $Z$ is 1-connected;

iv) $h^{0}\left(D-Z, \mathcal{O}_{D-Z}\right)=(D-Z) Z+p_{a}(Z)$.

2000 Mathematics Subject Classification. Primary: 14C20, Secondary: 14J99, $14 \mathrm{H} 45$.

Key words and phrases. dualizing sheaf, base component of canonical system, 1-connected curve, effective divisor on a surface.

The second named author is a member of the Center for Mathematical Analysis, Geometry and Dynamical Systems (IST-UTL). This work was partially supported by the Fundação para a Ciência e a Tecnologia through Program POCI 2010/FEDER and Grants-in-Aid for Scientific Research (B) (No. 16340008) by Japan Society for the Promotion of Science (JSPS). 
Furthermore if $p_{a}(Z)=0$ and $Z(D-Z)=m, D-Z$ decomposes as $B_{1}+\ldots+B_{m}$, such that $\mathcal{O}_{B_{i}}\left(-\left(B_{i+1}+\ldots+B_{m}\right)\right)=\mathcal{O}_{B_{i}}$, for every $i=1, \ldots, m-1, B_{i} Z=1$ and $B_{i}$ is 1-connected, for every $i=1, \ldots, m$. In addition either $B_{i} \preceq B_{i+1}+\ldots+B_{m}$ or $B_{i} \cap B_{i+1}+\ldots+B_{m}=\emptyset$.

\section{Preliminaries}

1.1. Notation. By a curve we mean a non-zero effective divisor on a smooth algebraic projective surface $S$ over $\mathbb{C}$. $K$ will denote the canonical bundle of $S$.

Given a curve $D, \omega_{D}$ denotes its dualizing sheaf and $p_{a}(D)$ its arithmetic genus.

A curve $D$ is $m$-connected if for every decomposition $D=A+B$, with $A, B$ effective non-zero divisors, $A B \geq m$.

For any invertible sheaf $\mathcal{L}$ on $D$ we denote by $h^{i}(D, \mathcal{L})$ the dimension as a $\mathbb{C}$-vector space of $H^{i}(D, \mathcal{L})$.

1.2. Some properties. Here we list some properties that will be used throughout without further reference.

- Given a curve $D, 2 p_{a}(D)-2=K D+D^{2}$ (adjunction formula).

- $h^{0}\left(D, \mathcal{O}_{D}\right)-h^{1}\left(D, \mathcal{O}_{D}\right)=1-p_{a}(D)$.

- By duality one has $h^{0}\left(D, \mathcal{O}_{D}\right)=h^{1}\left(D, \omega_{D}\right)$ and $h^{1}\left(D, \mathcal{O}_{D}\right)=$ $h^{0}\left(D, \omega_{D}\right)$.

- If a curve $D$ decomposes as the sum of two curves $D_{1}, D_{2}$ then $2 p_{a}(D)-2=K D+D^{2}=K D_{1}+K D_{2}+D_{1}^{2}+D_{2}^{2}+2 D_{1} D_{2}=$ $2 p_{a}\left(D_{1}\right)-2+2 p_{a}\left(D_{2}\right)-2+2 D_{1} D_{2}$ and so $p_{a}(D)=p_{a}\left(D_{1}\right)+$ $p_{a}\left(D_{2}\right)-1+D_{1} D_{2}$.

- ([CFM]) Let $D$ be an $m$-connected curve on the surface $S$ and let $D=D_{1}+D_{2}$ with $D_{1}, D_{2}$ curves. Then:

i) if $D_{1} \cdot D_{2}=m$, then $D_{1}$ and $D_{2}$ are $[(m+1) / 2]$-connected;

ii) if $D_{1}$ is chosen to be minimal subject to the condition $D_{1} \cdot\left(D-D_{1}\right)=m$, then $D_{1}$ is $[(m+3) / 2]$-connected.

\subsection{Auxiliary results.}

Definition 1.1. Let $D$ be a reducible curve on a smooth surface $S, \mathcal{L}$ an invertible sheaf on $D$ and let $s \in H^{0}(D, \mathcal{L})$ with $s \neq 0$ such that $s$ vanishes identically on some component of $D$. Let $Z_{s} \prec D$ be the biggest curve such that $s_{\mid Z_{s}} \equiv 0$. 
We will say that $s$ is 0 -maximal if there is no global section $t$ of $\mathcal{L}$ with $Z_{s} \prec Z_{t}$.

Lemma 1.2. Let $A$ be a curve such that $h^{0}\left(A, \mathcal{O}_{A}\right) \geq 2$. Then there is a decomposition $A=A_{1}+A_{2}$ where $A_{1}, A_{2}$ are curves such that

i) $h^{0}\left(A_{1}, \mathcal{O}_{A_{1}}\left(-A_{2}\right)\right) \neq 0$;

ii) $A_{1} A_{2} \leq 0$;

iii) for each component $\Gamma$ of $A_{1}, \Gamma A_{2} \leq 0$;

iv) for each component $\Gamma$ of $A_{1}$, the restriction map

$$
H^{0}\left(A_{1}, \mathcal{O}_{A_{1}}\left(-A_{2}\right)\right) \rightarrow H^{0}\left(\Gamma, \mathcal{O}_{\Gamma}\left(-A_{2}\right)\right)
$$

is injective;

v) for each component $\Gamma$ of $A_{1}$,

$$
h^{0}\left(A, \mathcal{O}_{A}\right) \leq h^{0}\left(A_{2}, \mathcal{O}_{A_{2}}\right)+h^{0}\left(\Gamma, \mathcal{O}_{\Gamma}\left(-A_{2}\right)\right) .
$$

Furthermore if $A_{1} A_{2}=0$ then $\mathcal{O}_{A_{1}}\left(-A_{2}\right)=\mathcal{O}_{A_{1}}$, and $h^{0}\left(A_{1}, \mathcal{O}_{A_{1}}\right)=1$.

Proof. Since $h^{0}\left(A, \mathcal{O}_{A}\right) \geq 2$, there exists a section $s$ in $H^{0}\left(A, \mathcal{O}_{A}\right)$ vanishing identically on some component of $A$. Choose a 0 -maximal such section $s$ and let $A_{2}:=Z_{s}, A_{1}:=D-A_{2}$. From the exact sequence

$$
0 \rightarrow \mathcal{O}_{A_{1}}\left(-A_{2}\right) \rightarrow \mathcal{O}_{A} \rightarrow \mathcal{O}_{A_{2}} \rightarrow 0
$$

we get

$$
0 \rightarrow H^{0}\left(A_{1}, \mathcal{O}_{A_{1}}\left(-A_{2}\right)\right) \rightarrow H^{0}\left(A, \mathcal{O}_{A}\right) \stackrel{r}{\rightarrow} H^{0}\left(A_{2}, \mathcal{O}_{A_{2}}\right) .
$$

By the hypothesis of 0-maximality of $s$, every section of $H^{0}\left(A_{1}, \mathcal{O}_{A_{1}}\left(-A_{2}\right)\right)$ does not vanish identically on any component $\Gamma$ of $A_{1}$ and so in particular $\Gamma A_{2} \leq 0$ and $A_{1} A_{2} \leq 0$. This proves assertions ii) and iii).

Furthermore for any $\Gamma$, again the hypothesis of 0 -maximality implies that the kernel of the restriction map

$$
H^{0}\left(A_{1}, \mathcal{O}_{A_{1}}\left(-A_{2}\right)\right) \rightarrow H^{0}\left(\Gamma, \mathcal{O}_{\Gamma}\left(-A_{2}\right)\right)
$$

is 0-dimensional and therefore we get assertions iv) and v), because

$$
h^{0}\left(A, \mathcal{O}_{A}\right) \leq h^{0}\left(A_{1}, \mathcal{O}_{A_{1}}\left(-A_{2}\right)\right)+h^{0}\left(A_{2}, \mathcal{O}_{A_{2}}\right) .
$$

The last assertion is clear, by the previous considerations. 
Remarks 1.3. a) In the decomposition above, if there is a component $\Gamma$ of $A_{1}$ such that $\Gamma A_{2}=0$, then $h^{0}\left(A_{1}, \mathcal{O}_{A_{1}}\left(-A_{2}\right)\right)=1$. Otherwise again one would get a contradiction to 0-maximality of $s$.

b) Lemma 1.2 means that a curve $D$ such that $h^{0}\left(D, \mathcal{O}_{D}\right) \geq 2$ is necessarily not 1 -connected. However $h^{0}\left(D, \mathcal{O}_{D}\right)=1$ does not imply 1connectedness. For instance a multiple fibre $F=m D$ of a fibration is not 1-connected but $h^{0}\left(F, \mathcal{O}_{F}\right)=1$ (cf. [BPV, Chp. III] )

Lemma 1.4. Let $D$ be a 1-connected curve. If $A \prec D$ is such that $A(D-A)=b$, then $h^{0}\left(A, \mathcal{O}_{A}\right) \leq b$.

Proof. We do this proof by induction on $b$. If $b=1$ then, because $D$ is 1-connected, $A$ is also and so $h^{0}\left(A, \mathcal{O}_{A}\right)=1$. We assume that we have proved the assertion for $m<b$ and we want to prove for $m=b$.

Suppose that $h^{0}\left(A, \mathcal{O}_{A}\right) \geq 2$. Take a decomposition of $A$ as in Lemma 1.2 and let $A_{1} A_{2}=-\alpha$, with $\alpha \geq 0$. Note that, because for every component $\Gamma$ of $A_{1}, \Gamma A_{2} \leq 0$, we have, for any component $\Gamma$ of $A_{1}, \Gamma A_{2} \geq-\alpha$, i.e. $\Gamma\left(-A_{2}\right) \leq \alpha$, and so $h^{0}\left(\Gamma, \mathcal{O}_{\Gamma}\left(-A_{2}\right)\right) \leq \alpha+1$.

Now, by 1-connectedness of $D, A_{1}\left(A_{2}+(D-A)\right) \geq 1$, and so $A_{1}(D-$ $A) \geq 1+\alpha$. Since $\left(A_{1}+A_{2}\right)(D-A)=b, A_{2}(D-A) \leq b-1-\alpha$ and so $A_{2}\left(A_{1}+(D-A)\right)=A_{2}\left(D-A_{2}\right) \leq b-1-2 \alpha$. Hence by the induction hypothesis $h^{0}\left(A_{2}, \mathcal{O}_{A_{2}}\right) \leq b-1-2 \alpha$ and so by Lemma 1.2 $h^{0}\left(A, \mathcal{O}_{A}\right) \leq b-\alpha$.

Corollary 1.5. Suppose that the curve $D$ is 1-connected. If $A \prec D$ is such that $A(D-A)=b$ and $h^{0}\left(A, \mathcal{O}_{A}\right)=b$, then $A$ decomposes as $B_{1}+\ldots+B_{b}$, such that $\mathcal{O}_{B_{i}}\left(-\left(B_{i+1}+\ldots .+B_{b}\right)\right)=\mathcal{O}_{B_{i}}$, for every $i=$ $1, \ldots, b-1, B_{i}(D-A)=1$ and $B_{i}$ is 1-connected, for every $i=1, \ldots, b$. Furthermore either $B_{i} \preceq B_{i+1}+\ldots .+B_{b}$ or $B_{i} \cap B_{i+1}+\ldots .+B_{b}=\emptyset$.

Proof. Suppose that $h^{0}\left(A, \mathcal{O}_{A}\right)=b$. Then in the decomposition as in the previous proof we must have $\alpha=0$ and $A_{1}(D-A)=1$, meaning that $A_{2}(D-A)=b-1$. So by Lemma 1.2 necessarily $h^{0}\left(A_{1}\left(-A_{2}\right)=1\right.$ and $\mathcal{O}_{A_{1}}\left(-A_{2}\right)=\mathcal{O}_{A_{1}}$.

Note also that $A_{1}\left(D-A_{1}\right)=1$ means that $A_{1}$ is 1-connected. Assume that $A_{1}$ has common components with $A_{2}$. Then we can write $A_{1}=$ $H+B, A_{2}=H+C$ where $B$ and $C$ have no common components and $H \neq 0$. Suppose $B \neq 0$. Since $\mathcal{O}_{A_{1}}\left(-A_{2}\right)=\mathcal{O}_{A_{1}}, B(H+C)=0$ and 
so, because $B C \geq 0$, we conclude that $B H \leq 0$. But this contradicts the 1-connectedness of $A_{1}$ and so $B=0$.

We take $B_{1}:=A_{1}$. Now we consider $D-A_{1}$ which is still 1-connected. One has $h^{0}\left(A_{2}, \mathcal{O}_{A_{2}}\right)=b-1$ and $A_{2}\left(D-A_{1}-A_{2}\right)=b-1$. We can apply the same reasoning as before and an obvious induction gives us the statement.

Lemma 1.6. Let $\mathcal{L}$ be an invertible sheaf on a curve $Z$ satisfying $\left.\operatorname{deg} \mathcal{L}\right|_{Z^{\prime}} \geq 2 p_{a}\left(Z^{\prime}\right)-2$ for any $0 \prec Z^{\prime} \preceq Z$. If $h^{1}(Z, \mathcal{L}) \neq 0$, then there exists a subcurve $A \preceq Z$ with $\left.\mathcal{L}\right|_{A} \simeq \omega_{A}$ and $h^{0}\left(A, \mathcal{O}_{A}\right)=1$.

Proof. By duality, we have $h^{0}\left(Z, \omega_{Z} \otimes \mathcal{L}^{-1}\right) \neq 0$. Take a 0 -maximal $s \in H^{0}\left(Z, \omega_{Z} \otimes \mathcal{L}^{-1}\right)$ and put $A=Z-Z_{s}$ (possibly $Z_{s}=0$ ). Then $\mathcal{O}_{A}\left(-Z_{s}\right) \otimes \omega_{Z} \otimes \mathcal{L}^{-1} \simeq \omega_{A} \otimes \mathcal{L}^{-1}$ and $s$ induces a non-zero $s^{\prime} \in$ $H^{0}\left(A, \omega_{A} \otimes \mathcal{L}^{-1}\right)$ which does not vanish identically on any component of $A$. In particular, $\omega_{A} \otimes \mathcal{L}^{-1}$ is nef. Since $\operatorname{deg} \omega_{A}=2 p_{a}(A)-2 \leq\left.\operatorname{deg} \mathcal{L}\right|_{A}$ by the assumption, $\omega_{A} \otimes \mathcal{L}^{-1}$ is numerically trivial. Furthermore, since $s^{\prime}$ is nowhere vanishing, we get $\omega_{A} \otimes \mathcal{L}^{-1} \simeq \mathcal{O}_{A}$. By the 0 -maximality of $s$, the restriction map $H^{0}\left(A, \omega_{A} \otimes \mathcal{L}^{-1}\right)=H^{0}\left(A, \mathcal{O}_{A}\right) \rightarrow H^{0}\left(\Gamma, \mathcal{O}_{\Gamma}\right)$ is injective for any irreducible component $\Gamma \preceq A$. Hence $h^{0}\left(A, \mathcal{O}_{A}\right)=$ 1.

Proposition 1.7. Let $Z$ be a curve such that for any $0 \prec Z^{\prime} \preceq Z$, $p_{a}\left(Z^{\prime}\right) \leq 0$. Then:

i) every component $\Gamma$ of $Z$ is a smooth rational curve;

ii) for any $0 \prec Z^{\prime} \preceq Z, h^{1}\left(Z^{\prime}, \mathcal{O}_{Z^{\prime}}\right)=0$.

Furthermore, $p_{a}(Z)=0\left(\right.$ and $\left.h^{0}\left(Z, \mathcal{O}_{Z}\right)=1\right)$ if and only if $Z$ is 1 connected.

Proof. By Lemma 1.6 applied to $\mathcal{L}=\mathcal{O}_{Z}$, if $h^{1}\left(Z, \mathcal{O}_{Z}\right) \neq 0$ there is a subcurve $A$ with $\mathcal{O}_{A} \simeq \omega_{A}$ and $h^{0}\left(A, \mathcal{O}_{A}\right)=1$. This implies $p_{a}(A)=1$ contradicting the hypothesis $p_{a}(A) \leq 0$. Therefore, $h^{1}\left(Z, \mathcal{O}_{Z}\right)=0$. Then we get i) and ii), since the natural map $H^{1}\left(Z, \mathcal{O}_{Z}\right) \rightarrow H^{1}\left(Z^{\prime}, \mathcal{O}_{Z^{\prime}}\right)$ is surjective for any $0 \prec Z^{\prime} \preceq Z$.

If $Z$ is 1 -connected, then $h^{0}\left(Z, \mathcal{O}_{Z}\right)=1$ (cf. Lemma 1.2) and we have $p_{a}(Z)=0$ by $h^{1}\left(Z, \mathcal{O}_{Z}\right)=0$. Conversely, assume that $p_{a}(Z)=0$. Then $0=p_{a}(Z)=p_{a}\left(Z_{1}\right)+p_{a}\left(Z_{2}\right)-1+Z_{1} Z_{2} \leq-1+Z_{1} Z_{2}$ for any decomposition $Z=Z_{1}+Z_{2}$ with $0 \prec Z_{1}, Z_{2}$. Hence $Z$ is 1 connected. 


\section{Fixed COMponents of $\left|\omega_{D}\right|$}

The results in this section prove Theorem 0.1 .

Proposition 2.1. Let $D$ be a 1-connected curve and $Z \prec D$ a curve such that the restriction map $H^{0}\left(D, \omega_{D}\right) \rightarrow H^{0}\left(Z, \omega_{D}\right)$ is the zero map. Then $p_{a}(Z) \leq 0$ and $h^{0}\left(D-Z, \mathcal{O}_{D-Z}\right)=(D-Z) Z+p_{a}(Z)$.

Proof. Let $B:=D-Z$. As usual one has $p_{a}(D)=p_{a}(B)+p_{a}(Z)-$ $1+B Z$, and so $p_{a}(B)-1=p_{a}(D)-B Z-p_{a}(Z)$.

Since the kernel of the restriction map $H^{0}\left(D, \omega_{D}\right) \rightarrow H^{0}\left(Z, \omega_{D}\right)$ is exactly $H^{0}\left(B, \omega_{B}\right)$, our hypothesis implies that $h^{0}\left(B, \omega_{B}\right)=h^{0}\left(D, \omega_{D}\right)$. The equality $p_{a}(B)-1=h^{0}\left(B, \omega_{B}\right)-h^{0}\left(B, \mathcal{O}_{B}\right)$ yields then

$$
p_{a}(D)-h^{0}\left(B, \mathcal{O}_{B}\right)=p_{a}(D)-B Z-p_{a}(Z),
$$

i.e., $h^{0}\left(B, \mathcal{O}_{B}\right)=B Z+p_{a}(Z)$.

By Lemma 1.4, $h^{0}\left(B, \mathcal{O}_{B}\right) \leq B Z$ and so $p_{a}(Z) \leq 0$.

Corollary 2.2. Let $D$ be a 1-connected curve and let $Z$ be the fixed part of $\left|\omega_{D}\right|$. Then for any $Z^{\prime} \preceq Z, p_{a}\left(Z^{\prime}\right) \leq 0$ and $h^{1}\left(Z^{\prime}, \mathcal{O}_{Z^{\prime}}\right)=0$.

Proof. The statement is an immediate consequence of Propositions 2.1 and 1.7 .

Corollary 2.3. Let $D$ be a 1-connected curve and $Z \prec D$ a 1-connected curve (or more generally such that $h^{0}\left(Z, \mathcal{O}_{Z}\right)=1$ ) such that the restriction map $H^{0}\left(D, \omega_{D}\right) \rightarrow H^{0}\left(Z, \omega_{D}\right)$ is the zero map. Then $h^{1}\left(Z, \mathcal{O}_{Z}\right)=$ 0 and $B:=D-Z$ decomposes as in Corollary 1.5.

Proof. By Corollary 2.2, $p_{a}(Z) \leq 0$ and $h^{1}\left(Z, \mathcal{O}_{Z}\right)=0$, and so, because $h^{0}\left(Z, \mathcal{O}_{Z}\right)=1$, we have $p_{a}(Z)=0$. By Proposition 2.1, $h^{0}\left(B, \mathcal{O}_{B}\right)=$ $B Z$ and therefore we can apply Corollary 1.5 obtaining a decomposition as wished.

\section{REFERENCES}

[BPV] W. Barth, C. Peters, A. Van De Ven, Compact complex surfaces, Ergebnisse der Mathematik und ihrer Grenzgebiete, 3. Folge, Band 4, SpringerVerlag, Berlin (1984).

[CCFR] F. Catanese, M. Franciosi, K. Hulek, M. Reid, Embeddings of curves and surfaces, Nagoya Math. J. 154 (1999), 185-220. 
[CFM] C. Ciliberto, P. Francia, M. Mendes Lopes, Remarks on the bicanonical map for surfaces of general type, Math. Z. 224 (1997), 137-166.

$[\mathrm{K}] \quad$ K. Konno, 1-2-3 theorem for curves on algebraic surfaces, J. Reine Angew. Math. 533 (2001), 171-205.

[M] M. Mendes Lopes, Adjoint systems on surfaces, Boll. Un. Mat. Ital. A (7) 10 (1996), no. 1, 169-179.

Department of Mathematics, Graduate School of Science, Osaka UNIVERSITY, TOYONAKA, OSAKA 560-0043, JAPAN

E-mail address: konno@math.sci.osaka-u.ac.jp

Center for Mathematical Analysis, Geometry, and Dynamical Systems, Departamento de Matemática, Instituto Superior Técnico, Universidade Técnica de Lisboa, Av. Rovisco Pais, 1049-001 Lisboa, PORTUGAL

E-mail address: mmlopes@math.ist.utl.pt 